\title{
Avaliação da Condição de Saúde Bucal e da Qualidade de Vida de Pacientes com Câncer de Cabeça e Pescoço Atendidos em um Hospital Público de Porto Alegre
}

\author{
Oral Health and Quality of Life Assessment of Patients with Head and Neck \\ Cancer treated at a Free Clinic in Porto Alegre
}

\author{
Silvânia Suely Caribé de Araúio', Dalva Maria Pereira Padilha², Julio Baldisserottoº
}

\begin{abstract}
Resumo
Este estudo teve por objetivo avaliar a Qualidade de Vida (QV) em pacientes com câncer de cabeça e pescoço, relacionado-a às condiçôes de saúde bucal. Foram pesquisados: variáveis socioeconômicas, hábitos e aspectos associados ao tumor, além de variáveis relacionadas à saúde bucal - índice de cárie composto por número de dentes cariados, perdidos e restaurados (CPOD), uso e necessidades protéticas, xerostomia e consulta ao dentista nos últimos seis meses, mucosite e mutilação. Foi utilizado o questionário de QV para câncer, QLQ-C30_Portuguese Brazilian, versão 3.0 e o questionário para pacientes com Câncer de cabeça e pescoço, EORTC QLQ - H\&N35_Portuguese Brazilian. A QV, no primeiro momento do estudo, foi considerada mediana, o nível funcional moderado e de baixa sintomatologia. Não houve correlação estatisticamente significativa entre o número de dentes perdidos e cariados com a QV. O grupo de pacientes que necessitava de próteses relatou desempenho funcional e função social menores. O grupo de pacientes com xerostomia demonstrou menor QV, menor nível funcional e maior sintomatologia. A QV dos pacientes no acompanhamento foi semelhante à primeira parte do estudo, porém houve aumento dos sintomas e diminuição do nível funcional. O CPOD aumentou e o uso de próteses decresceu, enquanto a necessidade das mesmas aumentou. Não foi possível verificar a associação entre a QV e a Mucosite, pois poucos pacientes foram afetados por essa condição. A mutilação foi correlacionada negativamente com o desempenho funcional, náusea e vômito, e perda de apetite. Apenas a avaliação clínica das variáveis de saúde bucal foi insuficiente para predizer a correlação entre a condição de saúde bucal e a QV, porém é essencial a atuação de uma equipe multidisciplinar de cuidado oncológico, na qual o cirurgião-dentista deve estar inserido de modo a minimizar os danos à saúde bucal desses pacientes, auxiliando na construção de uma melhor QV.
\end{abstract}

Palavras-chave: Qualidade de vida; Saúde bucal; Neoplasias de cabeça e pescoço; Epidemiologia

\footnotetext{
${ }^{1}$ Mestre em Saúde Bucal Coletiva pela Faculdade de Odontologia da Universidade Federal do Rio Grande do Sul (UFRGS). Professora do Departamento de Odontologia Restauradora da Faculdade de Odontologia da UFBA

${ }^{2}$ Professora da disciplina de Odontogeriatria do Departamento de Odontologia Preventiva e Social da Faculdade de Odontologia da UFRGS e Instituto de Geriatria e Gerontologia da PUCRS. Doutora em Estomatologia

${ }^{3}$ Professor Adjunto do Departamento de Odontologia Preventiva e Social da Faculdade de Odontologia da UFRGS. Doutor em Gerontologia Biomédica. Gerente de Ensino e Pesquisa do Grupo Hospitalar Conceiçāo

Este trabalho faz parte do Projeto Condição de Saúde Bucal e QV em Pacientes com Câncer de Cabeça e Pescoço atendidos no SUS, através do Edital: MCT/ SCTIE/DECIT/MS/CNPq no 038/2004 - Propostas sobre Saúde Bucal financiado pelo CNPq

Endereço para correspondência: Silvânia Suely Caribé de Araújo. Rua Edgard Reys Navarro, 376A - Apto 304. Santo Agostinho - Salvador (BA), Brasil - CEP: 40.255-280. E-mail: silvaniasuely@yahoo.com.br
} 


\section{INTRODUÇÃO}

A avaliação da Qualidade de Vida (QV) na Oncologia pode auxiliar na decisão sobre a efetividade do tratamento, melhorar a tomada de decisão do paciente através do esclarecimento dos efeitos colaterais do tratamento, servir como fator prognóstico para analisar os sintomas e/ou as necessidades de reabilitação, identificar os aspectos de impacto na sobrevida dos pacientes, a estimativa de custo-efetividade (auxilia na decisão de onde e quando investir os recursos existentes), melhorar a organização e a qualidade do cuidado, o desenvolvimento e a regulamentação de medicações, conhecer as prioridades dos pacientes ${ }^{1,2}$.

A função e a aparência da região de cabeça e pescoço são decisivas para a autoimagem e para a QV. Os bemestares físico, social e psicológico são bastante influenciados pela deformidade e pela disfunção decorrentes do câncer de cabeça e pescoço e seu tratamento ${ }^{3}$. Os tipos de tratamento de pacientes com câncer de cabeça e pescoço podem gerar prognósticos semelhantes, mas resultados diversos na QV desses pacientes. A QV deveria ser um fator a ser considerado no momento de escolha de um tratamento ${ }^{4}$.

A maioria dos estudos sobre QV relacionada à saúde utiliza variáveis sobre a doença, seus determinantes principais e seu tratamento. Em relação a pacientes com câncer do trato aerodigestivo superior, ainda não há uma definição clara da associação entre a condição de saúde bucal e a sua $\mathrm{QV}^{5}$. A função oral, entendida como um conjunto de processos que abrange a mastigação, a deglutição e a fonação, é apenas um recorte da QV relacionada à saúde, o qual foi abordado nesta pesquisa.

Conforme Rogers et al. ${ }^{6}$, há uma associação entre QV relacionada à saúde e função oral, logo, são fundamentais o conhecimento do impacto dessa associação e o planejamento adequado do tratamento da lesão cancerosa, de modo a minimizar os danos ao paciente. As principais complicações causadas pelo tratamento oncológico na cavidade bucal são: xerostomia, perda de paladar, hemorragia (afetando principalmente a mucosa labial, língua e gengiva), dermatite, mucosite, osteorradionecrose, trismo (espasmos musculares com ou sem fibrose dos músculos mastigatórios e da articulação temporomandibular) e mutilação ${ }^{7}$.

Os pacientes com câncer de cabeça e pescoço, além de estarem com uma doença que ameaça suas vidas, têm de lidar com o impacto dessa e de seu tratamento sobre aspectos funcionais e estéticos. Uma pesquisa feita com 75 pacientes tratados com cirurgia com ou sem radioterapia mostrou que, em seis meses do tratamento, houve piora da QV. Em 12 meses após o tratamento, houve uma melhora em todos os sintomas, porém a QV foi menor do que no período pré-tratamento. Pacientes com diferentes estágios da doença não apresentaram diferença significativa em sua QV; também não houve diferença entre os pacientes submetidos apenas à cirurgia e aqueles que tiveram tratamento cirúrgico e radioterápico, contudo, estes tiveram piores escores para sintomas físicos nos períodos de seis e 12 meses $^{8}$.

O objetivo principal deste estudo foi avaliar a QV em pacientes com câncer de cabeça e pescoço, relacionado-a às suas condições de saúde bucal.

\section{METODOLOGIA}

O delineamento deste estudo é quantitativo, observacional do tipo longitudinal, pois foram feitas duas avaliações prospectivas. A investigação foi realizada no setor de Oncologia do Hospital Nossa Senhora da Conceição (HNSC), em Porto Alegre, Rio Grande do Sul. A população-alvo foi constituída de 30 pacientes com o diagnóstico histopatológico de câncer nas regiōes de cabeça e pescoço. Critérios de inclusão: pacientes portadores de tumor primário e não submetidos a tratamento oncológico até o momento da primeira entrevista. O protocolo foi aprovado pelo Comitê de Ética em Pesquisa da Faculdade de Odontologia da Universidade Federal do Rio Grande do Sul, sob o ${ }^{\circ}$ 47/04 e pelo Comitê de Ética em Pesquisa do Grupo Hospitalar Conceição sob o n. 009/04.

A aplicação dos questionários e os exames clínicos odontológicos foram realizados em dois momentos distintos com intervalo de tempo de, no mínimo, seis meses entre eles. Foi utilizado o mesmo protocolo de tempo de estudos similares ${ }^{6}$. Os dados dos pacientes foram obtidos dos prontuários, constando de estadiamento do tumor, sua localização e tratamento realizado.

Os questionários utilizados foram o EORTC QLQC $30^{9}$ e EORTC QLQ-H\&N35․ O primeiro é genérico e o segundo específico para pacientes com câncer de cabeça e pescoço. Nos questionários, constam os seguintes domínios: saúde geral/QV, escalas funcional e de sintomas. A escala de sintomas é constituída dos seguintes itens: fadiga, náusea e vômito, dor, dispneia, insônia, perda de apetite, constipação, diarreia e dificuldades financeiras. A escala funcional é composta de função física, desempenho funcional, função emocional, cognitiva e social.

O exame de saúde bucal e a entrevista foram realizados por um único examinador. Foram utilizados os seguintes critérios para o exame de saúde bucal: a) para cárie: índice de dentes cariados, perdidos e 
obturados (CPOD) proposto pela Organização Mundial da Saúde $(\mathrm{OMS})^{10}$. Esse índice avalia a condição dentária em relação à variável cárie, tanto na coroa quanto na superfície radicular. Nesta pesquisa, não foram utilizados os códigos para tratamento. b) Condiçōes e Necessidades Protéticas: foram utilizados os códigos propostos pela $\mathrm{OMS}^{10}$. c) Para xerostomia, critérios de diagnóstico: avaliação subjetiva da função salivar que consta de quatro questôes categorizadas em sim e não. Para cada quatro repostas afirmativas, é caracterizada a redução do fluxo salivar em $1 \mathrm{~mL} / \mathrm{min}^{11}$; porém nesta pesquisa optou-se por considerar suspeita de xerostomia três respostas positivas. Optou-se por esse método subjetivo; pois, além de ser amparado na literatura, medidas objetivas do fluxo salivar demandariam um maior tempo de observação, sendo necessários dois momentos de um mesmo dia para medida, dificultando o processo de pesquisa.

As entrevistas duravam em média 40 minutos com cada paciente. No decorrer das entrevistas, a pesquisadora notou a dificuldade dos pacientes para responder às questôes e, então, foi criada uma escala visual de respostas baseada na escala do questionário e na escala de dor de faces e das cores da escala visual analógica. Segundo a Sociedade Brasileira para Estudo da Dor ${ }^{12}$, a escala de faces fornece, ao paciente, diversos rostos caracterizando uma gradação da dor e, do mesmo modo, a escala visual de cores apresenta uma progressão na intensidade das cores.

A primeira parte do estudo foi realizada no momento de admissão para tratamento e a segunda foi na fase pós-tratamento com um período mínimo de seis meses. Os questionários foram aplicados nas duas fases da pesquisa e, também, realizado um novo exame da cavidade bucal e avaliação para xerostomia.

As variáveis mucosite, mutilação pós-cirúrgica e tipo de tratamento oncológico foram coletadas nesta segunda parte do estudo. Foram utilizadas as seguintes fontes de coleta de dados de acordo com as variáveis: a) Para mucosite: relato do paciente e/ou diagnóstico médico/ odontológico. b) Para mutilação pós-cirúrgica: seguiramse os critérios de Baker ${ }^{13}$ : dissecção cervical radical, ressecção da bochecha com retalho reparador, parotidectomia total com dano ao nervo facial, laringectomia total, dissecção cervical bilateral, retirada da órbita ocular, hemimandibulectomia com dissecção cervical radical, amputação nasal, mandibulectomia parcial anterior, mandibulectomia segmental com dissecção cervical radical, retirada da órbita e maxilectomia radical. Nesta pesquisa, acrescentou-se glossectomia parcial e subtotal. c) Para o tipo de tratamento oncológico: esses dados foram obtidos através de pesquisa em documentos médicos que estavam em posse do paciente, pois apenas os tratamentos cirúrgico e quimioterápico eram oferecidos no HNSC, sendo a radioterapia realizada em outras instituições sem o acesso da pesquisadora.

\section{ANÁLISE ESTATÍSTICA}

Os dados foram analisados através do cálculo dos escores obtidos pela resposta a cada item dos questionários do estudo seguindo o manual da EORTC ${ }^{14}$. O programa estatístico utilizado foi o SPSS versão 8.0. A análise estatística foi realizada através do teste de correlação de Spearman e o teste $t$ de Student. Foram considerados os valores de $\mathrm{p}<0,05$ para as análises.

\section{RESULTADOS}

O perfil da amostra deste estudo foi composto de 25 homens; $67 \%$ dos pacientes examinados tinham entre 50 e 60 anos; e a média de idade foi de 59 anos. A maioria dos pacientes $(90 \%)$ cursou até o primeiro grau, sendo que 53,3\% recebiam até um salário mínimo. Em relação ao estado civil, 19 pacientes relataram viver com um(a) companheiro(a), 90\% dos entrevistados trabalhavam como autônomo ou estavam aposentados (Tabela 1).

Quanto aos hábitos no momento da entrevista, 53\% afirmaram que fumavam, $83 \%$ negaram consumir bebidas alcoólicas e $60 \%$ expuseram que não ingeriam chimarrão. A porcentagem de pacientes que bebiam e fumavam foi de $13 \%$. Considerando a história pregressa dos hábitos, 25 pacientes relataram ter fumado e 13 afirmaram ingerir bebidas alcoólicas.

$\mathrm{O}$ índice CPOD (que expressa a prevalência da doença cárie e suas sequelas) foi igual a 26,8 , sendo que o componente perdido foi responsável por $91,5 \%$ do CPOD, e o componente cariado, por $5,31 \%$. Vinte e três pacientes tinham mais de 20 dentes perdidos, sendo que 12 destes eram completamente edêntulos. $\mathrm{O}$ número de dentes cariados ou restaurados variou de zero a dez; sete pessoas possuíam dentes cariados, três possuíam dentes restaurados e quatro possuíam dentes cariados e restaurados.

Quarenta e três por cento dos pacientes usavam prótese total superior e $23,3 \%$ usavam prótese total inferior. Em relação às necessidades protéticas, $20 \%$ dos pesquisados necessitavam de prótese total superior e $17 \%$ necessitavam de uma associação de próteses unitárias e/ou com múltiplos elementos na arcada superior; $46,7 \%$ dos pacientes necessitavam de uma associação de próteses unitárias e/ou com múltiplos elementos na arcada inferior e $20 \%$ necessitavam de prótese total inferior. Dezenove $(63,3 \%)$ pacientes 
Tabela 1. Perfil socioeconômico da amostra estudada $(n=30)$. Porto Alegre, RS, 2005

\begin{tabular}{|c|c|c|c|}
\hline Variáveis & Categoria & $\begin{array}{l}\mathbf{N} \\
(\%)\end{array}$ & $\begin{array}{c}\text { Média } \\
\text { (extensão) }\end{array}$ \\
\hline \multirow{4}{*}{ Sexo } & Mulher & $5(16,7 \%)$ & \\
\hline & Homem & $25(83,3 \%)$ & \\
\hline & $<50$ anos & $3(10 \%)$ & \\
\hline & 50 a 60 anos & $20(66,7 \%)$ & \\
\hline \multirow[t]{5}{*}{ Faixa etária } & 61 a 70 anos & $4(13,3 \%)$ & $59(44-86)$ \\
\hline & 71 a 80 anos & $2(6,7 \%)$ & \\
\hline & 81 a 90 anos & $1(3,3 \%)$ & \\
\hline & Analfabeto(a) & $1(3,3 \%)$ & \\
\hline & Semianalfabeto(a) & $2(6,7 \%)$ & \\
\hline \multirow[t]{4}{*}{ Escolaridade } & $1^{\circ}$ grau & $24(80 \%)$ & \\
\hline & $2^{\circ}$ grau & $2(6,7 \%)$ & \\
\hline & $3^{\circ}$ grau incompleto & $1(3,3 \%)$ & \\
\hline & < 1salário mínimo & $4(13,3 \%)$ & \\
\hline \multirow{4}{*}{ Renda } & 1 salário mínimo & $12(40 \%)$ & \\
\hline & 1 a 5 salários mínimos & $13(43,3 \%)$ & \\
\hline & 6 a 10 salários mínimos & $1(3,3 \%)$ & \\
\hline & Possui companheiro(a) & $19(63,3 \%)$ & \\
\hline \multirow{3}{*}{ Estado civil } & Solteiro(a) & $1(3,3 \%)$ & \\
\hline & Divorciado(a) & $7(23,3 \%)$ & \\
\hline & Viúvo(a) & $3(10 \%)$ & \\
\hline \multirow{4}{*}{ Ocupação } & Aposentado(a) & $12(40 \%)$ & \\
\hline & Desempregado(a) & $2(6,7 \%)$ & \\
\hline & Autônomo(a) & $15(50 \%)$ & \\
\hline & Trabalhador(a) formal & $1(3,3 \%)$ & \\
\hline
\end{tabular}

queixaram-se de xerostomia. Apenas oito (26,7\%) pessoas realizaram consulta odontológica nos últimos seis meses. A média da QV da amostra estudada foi de $52(+19,7)$, sendo que a QV é quantificada através de uma escala que varia de zero a 100 e esse valor não possui unidade de medida. A mediana da QV foi 50 , sendo que os valores máximo e mínimo alcançados foram de 92 e zero, respectivamente. Com relação às escalas funcional e de sintomas, os valores medidos estão representados na Tabela 2.

Com relação à variável CPOD, foram feitas correlaçôes entre os componentes perdido e cariado do CPOD, pois representam um maior impacto na saúde bucal, e as variáveis $\mathrm{QV}$, escala funcional e escala de sintomas. As correlações entre essas variáveis (Tabela 3) foram fracas, sem significância estatística, ou inexistentes. A amostra foi dividida por grupos - de acordo com a necessidade de próteses, hábitos e queixa de xerostomia - para facilitar a análise de características que podem influenciar na QV. O grupo de pacientes que não realizou consulta odontológica, nos últimos seis meses, foi pequeno (oito pacientes), o que dificultou as análises estatísticas através de teste de correlação ou teste $t$ de Student entre os grupos que realizaram ou não consulta odontológica nos últimos seis meses. Também não foi analisada a $\mathrm{QV}$ daqueles que usavam ou não prótese, pois não foram feitos testes de qualidade da prótese e nem da eficiência mastigatória, as quais poderiam servir de variáveis de confundimento.

Vinte $(66,7 \%)$ pacientes da amostra necessitavam de algum tipo de prótese tanto na arcada superior quanto na inferior. Para o grupo de pacientes que necessitavam de prótese, os escores de QV foram levemente maiores do que no grupo daqueles que não necessitavam de prótese, a função física foi semelhante nos dois grupos. O desempenho funcional e a função social foram menores, mas as funções emocional e cognitiva foram maiores no grupo que necessitava de prótese. Não houve diferenças estatisticamente significantes na comparação das médias nos dois grupos mencionados quanto à $\mathrm{QV}$ e escala funcional. $\mathrm{O}$ grupo de pacientes com xerostomia $(n=19)$ demonstrou menor QV, menor nível funcional e maior sintomatologia quando comparado com aqueles sem queixa de xerostomia $(n=11)$. Houve significância estatística para as variáveis dispneia e perda de apetite, ambas componentes da escala de sintomas. 
Tabela 2. Valores das escalas funcional e de sintomas nos pacientes pesquisados $(n=30)$. Porto Alegre, RS, 2005

\begin{tabular}{c|c|c|c|c|c}
\hline & Média & Desvio-padrão & Mediana & Máximo & Mínimo \\
\hline QV & 52 & 19,7 & 50 & 91,7 & 0 \\
Função Física & 77 & 20,6 & 80 & 100 & 40 \\
Desempenho Funcional & 61 & 34,9 & 58 & 100 & 0 \\
Função Emocional & 50 & 30,9 & 54 & 100 & 0 \\
Função Cognitiva & 73 & 28,6 & 83 & 100 & 17 \\
Função Social & 74 & 36 & 100 & 100 & 0 \\
Fadiga & 29 & 25 & 0 & 78 & 0 \\
Náusea e Vômito & 12 & 18,5 & 0 & 50 & 0 \\
Dor & 46 & 37,1 & 0 & 100 & 0 \\
Dispneia & 26 & 33,5 & 0 & 100 & 0 \\
Insônia & 28 & 34 & 0 & 100 & 0 \\
Perda de Apetite & 17 & 32,5 & 33 & 100 & 0 \\
Constipação & 39 & 43 & 0 & 33 & 0 \\
Diarreia & 4 & 11,5 & 33 & 100 & 0 \\
\hline Dificuldades Financeiras & 43 & 41,2 & & 0 & 0 \\
\hline
\end{tabular}

Tabela 3. Coeficientes de Correlação de Spearman e os valores de $p$ entre as variáveis cariado e perdido, QV e as variáveis das Escalas Funcional e de Sintomas nos pacientes $(n=30)$. Porto Alegre, RS, 2005

\begin{tabular}{c|c|c}
\hline Variáveis & $\begin{array}{c}\text { Cariado } \\
\text { Coeficiente de correlação }(\mathbf{p})\end{array}$ & $\begin{array}{c}\text { Perdido } \\
\text { Coeficiente de correlação }(\mathbf{p})\end{array}$ \\
\hline QV & $-0,264(0,159)$ & $-0,103(0,589)$ \\
Função Física & $0,049(0,795)$ & $-0,255(0,173)$ \\
Desempenho Funcional & $-0,124(0,513)$ & $-0,013(0,944)$ \\
Função Emocional & $0,037(0,847)$ & $0,058(0,760)$ \\
Função Cognitiva & $0,358(0,520)$ & $-0,121(0,524)$ \\
Função Social & $0,003(0,989)$ & $0,124(0,512)$ \\
Fadiga & $0,045(0,815)$ & $-0,056(0,767)$ \\
Náusea e Vômito & $0,123(0,516)$ & $-0,155(0,413)$ \\
Dor & $0,108(0,571)$ & $0,056(0,767)$ \\
Dispneia & $-0,081(0,669)$ & $-0,80(0,673)$ \\
Insônia & $0,124(0,514)$ & $-0,304(0,103)$ \\
Perda de Apetite & $0,163(0,397)$ & $0,032(0,871)$ \\
Constipação & $-0,032(0,868)$ & $-0,032(0,865)$ \\
Diarreia & $-0,138(0,468)$ & $0,029(0,878)$ \\
Dificuldades Financeiras & $-0,295(0,114)$ & $0,135(0,478)$ \\
\hline
\end{tabular}

Observando o grupo de fumantes $(\mathrm{n}=16)$ com relação ao grupo dos não fumantes $(\mathrm{n}=14)$, aquele grupo apresentou valores médios maiores de QV - relativa apenas à percepção que consta nas questôes 29 e 30 do questionário, porém esse grupo possui menor QV no geral -, função física, fadiga, náusea e vômito, dor, dispneia, insônia, perda de apetite e constipação. No grupo dos não fumantes, os maiores escores médios foram para desempenho funcional, função emocional, função cognitiva, diarreia e dificuldades financeiras. $\mathrm{O}$ grupo dos fumantes mostrou um maior prejuízo na escala funcional e um maior impacto, quanto aos sintomas, do que o grupo dos não fumantes, apesar do primeiro grupo relatar uma maior $\mathrm{QV}$, porém houve significância estatística para a variável dor.

Cinco pacientes afirmaram ingerir bebidas alcoólicas no momento da entrevista, sendo este um número muito pequeno de indivíduos para serem feitas comparaçōes com o grupo daqueles que não ingeriam bebidas alcoólicas. Os indivíduos pesquisados que ingeriam chimarrão $(\mathrm{n}=12)$ apresentaram maior $\mathrm{QV}$, porém menor nível funcional e maior sintomatologia do que aqueles que não ingeriam chimarrão $(n=18)$, entretanto essas diferenças entre as médias não foram estatisticamente significantes. 
A amostra na segunda parte do estudo foi reduzida à metade, sendo que dez pacientes foram a óbito antes da visita domiciliar e cinco pacientes não foram encontrados. O perfil desse grupo de pacientes $(n=15)$ foi maioria do sexo masculino $(86,7 \%)$, na faixa etária de 50 a 60 anos (80\%), com média de idade de 57,8 anos. O grau de escolaridade foi o primeiro grau $(86,7 \%)$, a renda foi de um a cinco salários mínimos para $60 \%$ da amostra, de um salário mínimo para 33,3\%, e uma pessoa $(6,7 \%)$ recebia menos de um salário mínimo. Sessenta por cento dos pesquisados(as) vivem com um(a) companheiro(a), 46,7\% trabalham como autônomos e 40\% são aposentados. Quatro pacientes realizaram consulta odontológica nos últimos seis meses. Quanto à saúde bucal desses pacientes, dois pacientes apresentaram perdas dentárias após o primeiro exame e entrevista, dois pacientes tiveram um aumento no número de dentes afetados por cárie e dois mostraram um incremento na perda dentária e no número de dentes cariados. O CPOD foi de 27,7 nesta segunda parte do estudo, sendo que na primeira parte foi de 26,8. Um dos pacientes diminuiu o seu CPOD, pois no momento do segundo exame mostrou um pré-molar inferior hígido recém-erupcionado, o qual estava incluso no momento do primeiro exame clínico.

A necessidade do uso de próteses aumentou nos pacientes, comparando-se os dois momentos do estudo, já que na segunda fase da pesquisa os pacientes não estavam usando suas próteses ou permaneciam com a necessidade apresentada no primeiro momento. Quatro pacientes permaneceram fumando, um paciente afirmou ingerir bebidas alcoólicas e seis pessoas continuaram a ingerir chimarrão. $O$ tratamento realizado foi a combinação de cirurgia e radioterapia em 11 pacientes, dois foram submetidos à cirurgia, um foi tratado com quimioterapia e radioterapia, e um paciente decidiu não realizar nenhum tratamento. Cinco pacientes relataram terem sido afetados pela mucosite, seis queixaram-se de xerostomia (ambos os grupos foram tratados com cirurgia e radioterapia) e sete sofreram algum tipo de mutilação. $\mathrm{O}$ fato de alguns pacientes apresentarem mudança na xerostomia de presença (sim) para ausência (não) na primeira para a segunda parte do estudo pode ser explicado pela limitação do questionário utilizado que contempla quatro itens, então, se o paciente apresentava duas queixas de xerostomia, isso não era aceitável de acordo com o questionário, que considerava xerostomia quando eram respostas afirmativas para todos os itens.

A média da QV, tanto da primeira quanto da segunda parte do estudo, foi semelhante, não apresentando correlação estatisticamente significante. Com relação à escala funcional, a função física, o desempenho funcional e a função social decresceram; mas a função cognitiva aumentou, apresentando uma correlação significante, com coeficiente de correlação de grau moderado. A média da função emocional permaneceu sem alterações nas duas etapas da pesquisa.

A sintomatologia agravou-se na segunda parte do estudo quando comparada com a primeira, todas as variáveis da escala de sintomas mostraram aumento de suas médias. Houve correlações significantes para as variáveis dispneia e insônia (significante a 0,01 ). Os graus dos coeficientes de correlação para a dispneia e insônia entre si foram fortes (Tabela 4).

Não foram realizados testes de correlação ou teste $t$ de Student entre os diferentes tipos de tratamento e ausência do mesmo devido ao pequeno número de pessoas em cada grupo de tratamento, sendo que no grupo de não-tratamento havia uma pessoa. Por essas mesmas razões, não foram feitas as análises estatísticas para o grupo de pacientes que afirmou ter realizado consulta odontológica nos últimos seis meses (quatro pessoas), ter sido afetado por mucosite e por xerostomia (cinco e seis pacientes, respectivamente), além de que a associação entre mucosite e QV seria mais bem verificada no período suscetível de desenvolvimento dessa lesão (uma a duas semanas após início da radioterapia ou quimioterapia). Verificou-se, porém, a correlação entre QV e a variável mutilação, já que $46,7 \%$ dos pacientes $(n=7)$ foram afetados por esta condição. A mutilação foi correlacionada negativamente com o desempenho funcional (forte grau de correlação significante a 0,001$)$.

\section{DISCUSSÃO}

A amostra deste estudo constou de 30 pacientes com câncer de cabeça e pescoço atendidos no ambulatório de Oncologia do HNSC, nos períodos de maio a agosto de 2004. Essa amostra condiz com a média mensal de 36 pacientes tratados para cabeça e pescoço no ano de 2001 nos seis centros de tratamento oncológico do Sistema Único de Saúde (SUS) em Porto Alegre-RS, Centro de Alta Complexidade em Oncologia (CACON $)^{15}$.

A maioria dos pacientes era do sexo masculino, com uma média de idade de 59 anos. O câncer de cabeça e pescoço é mais prevalente no sexo masculino e na faixa etária acima dos $50 \operatorname{anos}^{16}$. No momento da primeira entrevista, $83 \%$ dos pacientes relataram história passada do hábito de fumar e $43 \%$ do hábito de ingerir bebidas alcoólicas. Mais da metade dos pacientes fumava, 16,7\% consumiam bebidas alcoólicas e 13\% afirmaram beber e fumar na entrevista inicial. Isso indica a manutenção 
Tabela 4. Médias, desvios-padrões e medianas das variáveis $Q V$ e da escala funcional e de sintomas nos pacientes pesquisados ( $n=15$ ). Coeficientes de correlação de Spearman e os valores de $p$ entre as variáveis da escala funcional. Porto Alegre, RS, 2005

\begin{tabular}{|c|c|c|c|c|c|}
\hline \multirow[b]{2}{*}{ Variáveis } & \multicolumn{2}{|c|}{ Parte I } & \multicolumn{2}{|c|}{ Parte II } & \multirow{2}{*}{$\begin{array}{c}\text { Coeficiente } \\
\text { de } \\
\text { Correlação } \\
\text { (p) }\end{array}$} \\
\hline & $\begin{array}{l}\text { Média } \\
\text { (desvio- } \\
\text { padrão) }\end{array}$ & Mediana & $\begin{array}{l}\text { Média } \\
\text { (desvio- } \\
\text { padrão) }\end{array}$ & Mediana & \\
\hline QV & $\begin{array}{c}55,5 \\
(19,2)\end{array}$ & 58,3 & $\begin{array}{c}55 \\
(21,5)\end{array}$ & 58,3 & $\begin{array}{c}0,124 \\
(0,660)\end{array}$ \\
\hline Função Física & $\begin{array}{c}75,6 \\
(21,3)\end{array}$ & 73,3 & $\begin{array}{c}53,3 \\
(34,1)\end{array}$ & 60 & $\begin{array}{c}0,447 \\
(0,095)\end{array}$ \\
\hline Desempenho Funcional & $\begin{array}{l}60,0 \\
(32)\end{array}$ & 66,7 & $\begin{array}{c}51,1 \\
(41,9)\end{array}$ & 50 & $\begin{array}{c}0,272 \\
(0,326)\end{array}$ \\
\hline Função Emocional & $\begin{array}{c}58,9 \\
(26,3)\end{array}$ & 58,3 & $\begin{array}{c}58,9 \\
(28,9)\end{array}$ & 58,3 & $\begin{array}{c}0,280 \\
(0,311)\end{array}$ \\
\hline Função Cognitiva & $\begin{array}{c}71,1 \\
(31,2)\end{array}$ & 83,3 & $\begin{array}{c}74,5 \\
(33,3)\end{array}$ & 100 & $\begin{array}{c}0,522 \\
(0,046)\end{array}$ \\
\hline Função Social & $\begin{array}{c}74,4 \\
(32,1)\end{array}$ & 83,3 & $\begin{array}{c}65,5 \\
(33,0)\end{array}$ & 66,7 & $\begin{array}{c}0,462 \\
(0,083)\end{array}$ \\
\hline Fadiga & $\begin{array}{c}25,9 \\
(22,2)\end{array}$ & 22,2 & $\begin{array}{c}40,7 \\
(37,7)\end{array}$ & 33,3 & $\begin{array}{c}0,287 \\
(0,300)\end{array}$ \\
\hline Náusea e Vômito & $\begin{array}{c}8,9 \\
(15,3)\end{array}$ & 0 & $\begin{array}{c}15,6 \\
(26,3)\end{array}$ & 0 & $\begin{array}{c}0,421 \\
(0,118)\end{array}$ \\
\hline Dor & $\begin{array}{c}35,6 \\
(37,2)\end{array}$ & 16,7 & $\begin{array}{c}47,8 \\
(44,5)\end{array}$ & 50 & $\begin{array}{c}0,281 \\
(0,310)\end{array}$ \\
\hline Dispnéia & $\begin{array}{c}24,4 \\
(29,5)\end{array}$ & 33,3 & $\begin{array}{c}28,9 \\
(37,6)\end{array}$ & 0 & $\begin{array}{c}0,605 \\
(0,017)\end{array}$ \\
\hline Insônia & $\begin{array}{c}20 \\
(30,3)\end{array}$ & 0 & $\begin{array}{c}42,2 \\
(46,3)\end{array}$ & 33,3 & $\begin{array}{l}0,824^{*} \\
(0,000)\end{array}$ \\
\hline Perda de Apetite & $\begin{array}{c}13,3 \\
(27,6)\end{array}$ & 0 & $\begin{array}{c}42,2 \\
(47,9)\end{array}$ & 0 & $\begin{array}{c}0,086 \\
(0,761)\end{array}$ \\
\hline Constipação & $\begin{array}{c}40 \\
(42,2)\end{array}$ & 33,3 & $\begin{array}{c}28,9 \\
(37,5)\end{array}$ & 0 & $\begin{array}{c}0,371 \\
(0,174)\end{array}$ \\
\hline Diarreia & $\begin{array}{c}2,2 \\
(8,6)\end{array}$ & 0 & $\begin{array}{c}8,9 \\
(26,6)\end{array}$ & 0 & $\begin{array}{r}-0,092 \\
(0,744)\end{array}$ \\
\hline Dificuldades Financeiras & $\begin{array}{c}37,8 \\
(37,6)\end{array}$ & 33,3 & $\begin{array}{c}46,7 \\
(41,5)\end{array}$ & 37,3 & $\begin{array}{r}-0,346 \\
(0,207)\end{array}$ \\
\hline
\end{tabular}

Significância estatística em negrito.

*Correlação significante no nível de $\mathrm{p}<0,01$.

de hábitos que são considerados fatores de risco para o desenvolvimento do câncer de cabeça e pescoço, podendo influir negativamente no tratamento oncológico ${ }^{17}$.

Aproximadamente $63 \%$ dos pacientes avaliados queixaram-se de xerostomia no momento da primeira entrevista. Nicolatou-Galitis et al..$^{18}$ afirmaram que a xerostomia pode ser influenciada pelo estado psicológico e somático e pelo estado de hidratação do paciente, entre outros fatores. Dos 30 pacientes pesquisados, 22 não realizaram consulta odontológica nos últimos seis meses, sendo que o cirurgião-dentista não participou do processo diagnóstico. Sabe-se que o campo de atuação do cirurgião-dentista é a cavidade bucal; porém, como profissional de saúde, o cirurgião-dentista deveria observar as alteraçôes e/ou queixas do paciente de modo integral e encaminhá-lo ao profissional especialista. Esse fato pode ser explicado pela dificuldade de acesso da maioria da população brasileira ao Serviço de Saúde Bucal (SB). Dados do SB Brasil ${ }^{19}$ sobre acesso a serviços odontológicos mostraram que, na faixa etária de 65 a 74 anos, 5,83\% nunca tinham ido ao dentista, 16,83\% tinham se consultado há menos de um ano e 65,69\% há mais de três anos; $0,58 \%$ se consultou por causa de feridas, caroços ou manchas na boca, que podem ser sinais precoces do câncer bucal ${ }^{16}$. O motivo prevalente de consulta odontológica foi dor $(48,12 \%)$; porém o câncer, em seus estágios iniciais, é geralmente indolor ${ }^{16}$. 
A média da QV dos pacientes estudados foi 52, menor do que a média relatada para pacientes com câncer de cabeça e pescoço no manual de valores de referência do questionário EORTC QLQ-C30, que foi $70^{8}$. Os itens da escala funcional tiveram médias de 50 a 77, com menor média para a função emocional (50), e a maior para a função física. Embora essas médias tenham sido menores do que as referidas no manual (variação entre 85,1 a 86,4), houve uma semelhança na distribuição das médias das variáveis: menor média para a função emocional (70) e maior para a função física $(86,4)$. A sintomatologia foi maior na amostra pesquisada do que nos pacientes do manual; as maiores diferenças foram entre a dor ( 46 e 22,5, respectivamente), dispneia (26 e 16,6, respectivamente), constipação (39 e 8,1, respectivamente) e dificuldades financeiras (43 e 11,4, respectivamente). Entretanto, deve-se atentar para as diferenças entre as populaçóes das quais as amostras se originam: Brasil (presente estudo) e Holanda, Suécia e Noruega (amostra do manual de valores de referência ${ }^{20}$ ).

A avaliação da QV nos pacientes que necessitam de prótese demonstrou que não houve diferenças estatisticamente significativas entre a QV e escala funcional dos grupos que necessitam ou não de prótese, apesar do desempenho funcional e da função social serem menores no grupo de pacientes que necessitam de prótese. De acordo com Allison, Locker e Feine ${ }^{5}$, as pessoas totalmente edêntulas e que não usam prótese estariam mais preparadas para enfrentar os transtornos decorrentes do tratamento do câncer, devido à superação de problemas anteriores, como a adaptação às perdas dentárias. Esses dados estão coerentes com os resultados alcançados, apesar de somente quatro pacientes do grupo que necessita de próteses serem completamente edêntulos.

O grupo de pacientes com xerostomia obteve menor $\mathrm{QV}$ e maiores dificuldades funcionais e sintomas do que o grupo sem xerostomia. O primeiro grupo apresentou mais dispneia e perda de apetite do que o segundo, sendo essas diferenças significativas. A xerostomia afeta negativamente a QV. Segundo Eisbruch $^{21}$, a xerostomia em pacientes com câncer é prevalente antes do tratamento radioterápico; essa condição provoca dificuldade de mastigação e deglutição, e aumenta a suscetibilidade a lesôes na mucosa bucal.

Os pacientes fumantes tiveram uma maior QV; porém menor nível funcional e mais sintomas, principalmente dor, a qual foi estatisticamente significativa. Com relação ao resultado da $\mathrm{QV}$ no grupo de fumantes, é necessário lembrar que a QV é subjetiva; portanto avaliada pelo próprio paciente, podendo não condizer com a real QV que leva em conta outras variáveis (escalas funcional e de sintomas) e não apenas as questões 29 e 30 que dizem respeito à percepção do paciente sobre sua saúde geral e $\mathrm{QV}^{14}$. A ingestão de chimarrão não causou impacto significativo sobre a $\mathrm{QV}$ dos pacientes examinados; porém a literatura carece de informações sobre esse dado.

$O$ estudo não demonstrou correlação significativa entre QV e dentes perdidos ou cariados. Sugere-se que isso não foi demonstrado devido ao uso de estratégias de coping em relação à saúde bucal, as quais são influenciadas por características individuais e coletivas, como a cultura. Para Chen, Harmon e Andersen ${ }^{22}$, a percepção e o relato de sintomas e condiçôes bucais são influenciados pelos níveis de tolerância e sensibilidade aceitos pelo indivíduo e pela sociedade na qual ele está inserido; a comunidade onde a pessoa reside exerce forte influência sobre a adaptabilidade individual às doenças bucais e suas consequências.

Além disso, para a maioria das pessoas, as condições bucais não alteram o curso de suas vidas, e nem trazem sérias incapacidades, apesar de gerar um impacto sobre a vida diária. Sugere-se o uso de instrumentos específicos que meçam a QV relacionada à saúde bucal, pois a avaliação de variáveis clínicas é limitada.

$\mathrm{Na}$ segunda parte do estudo, o CPOD de 66,7\% dos 15 pacientes pesquisados não sofreu alterações entre os dois exames realizados. Um estudo realizado por Allison, Locker e Feine ${ }^{5}$ mostrou que $81,4 \%$ dos pacientes tratados de câncer do trato aerodigestivo superior não tiveram mudança no estado dentário após o diagnóstico e tratamento do tumor.

Com relação ao tipo de tratamento oncológico, a maioria dos pacientes foi tratada com cirurgia e radioterapia. A QV no acompanhamento dos pacientes foi semelhante à da primeira parte da pesquisa, houve uma correlação significativa entre a função cognitiva dos dois períodos, a qual aumentou. Houve uma diminuição do nível funcional geral nesta segunda parte do estudo, e também um aumento da sintomatologia, especificamente dispneia e insônia. A QV esteve correlacionada com o desempenho funcional e com as funções emocional e cognitiva. A função física foi associada com o desempenho funcional, e a função cognitiva com as funçôes emocional e social. Rogers ${ }^{23}$ relataram um aumento não significativo da $\mathrm{QV}$ no período pós-operatório de seis meses em pacientes tratados de câncer bucal; os pacientes com menores danos na fonação apresentaram uma melhor QV.

Para avaliação da mucosite, foi perguntado ao paciente se ele apresentou alguma ferida dolorosa na boca, diagnosticada ou não, na época da radioterapia ou da quimioterapia. A avaliação subjetiva de mucosite foi realizada, pois, de acordo com Rose-Ped et al. ${ }^{24}$, as 
sequelas dolorosas foram consideradas pelos pacientes como a pior parte do tratamento oncológico. "Sequelas dolorosas são memoráveis" (tradução nossa) ${ }^{24}$ (p. 464). Cinco pacientes relataram apresentar mucosite durante a radioterapia, mas como não foi possível a realização da entrevista no período suscetível de mucosite, as análises estatísticas não foram realizadas; porém há comprovação na literatura de que a mucosite causa dor intensa, distúrbios no paladar, entre outras, comprometendo a mastigação, deglutição e fonação; enfim, causando prejuízo à $\mathrm{QV}$ dos pacientes afetados por essa condição patológica ${ }^{7}$.

Metade dos pacientes que se queixaram de xerostomia pós-tratamento tinha sido submetida à radioterapia. Esse resultado foi semelhante ao estudo de Bjordal et al..$^{25}$, em que $50 \%$ dos pacientes com câncer de cabeça e pescoço tratados com radioterapia apresentaram sintomas de xerostomia no período de um a seis anos após o início do tratamento. Devido ao pequeno número de indivíduos que apresentaram xerostomia, não foram feitas análises estatísticas entre essa variável e a QV.

Em relação ao fumo, aproximadamente $73 \%$ dos 15 pacientes pesquisados não continuaram a fumar; esses dados são opostos aos de Bjordal et al..$^{25}$, em que $22 \%$ de 50 pacientes pararam de fumar após o tratamento. Esses autores indicam a implementação de programas que auxiliem o paciente a parar de fumar. Para Baker ${ }^{13}$, a continuidade de hábitos nocivos como fumo e ingestão de álcool representa barreiras para a reabilitação de pacientes com câncer de cabeça e pescoço. A mutilação esteve presente em sete pacientes e foi correlacionada significativamente com o desempenho funcional e com a perda de apetite. Para Rogers ${ }^{23}$, a extensão e o local das ressecçôes de tecidos moles nas cirurgias orais de remoção de câncer têm um impacto significativo sobre a função pós-operatória.

\section{CONCLUSÃO}

Todos os profissionais de saúde da equipe oncológica, inclusive o cirurgião-dentista, deveriam ser capacitados para aconselhamento informal dos pacientes e para encaminhá-los ao especialista quando necessário. A literatura é unânime em afirmar o papel de uma equipe de saúde multidisciplinar no cuidado e suporte ao paciente oncológico e sua família. É essencial a investigação da QV de pacientes com câncer de cabeça e pescoço, a qual deve ser incorporada à prática clínica, devido à sua utilidade no processo de decisão de tratamento, controle das sequelas, instituição de medidas preventivas, enfim, melhoria da sobrevida desses pacientes ${ }^{2,4}$. Observou-se nesta pesquisa a necessidade que o paciente com câncer de cabeça e pescoço e seus familiares têm de serem ouvidos e acompanhados em um momento que é de sofrimento e perplexidade frente ao diagnóstico da doença, e no processo de aprender a lidar com as condições adversas do tratamento, controlando ou evitando-as. A saúde bucal é um elemento fundamental do cuidado oncológico que deve ser buscada e preservada de modo a contribuir para a manutenção da QV do paciente. É importante que a equipe oncológica multidisciplinar exerça um cuidado oncológico mais humanizado: ouvindo, orientando, adequando protocolos e amparando o enfermo e seus familiares. A formação de vínculos entre profissionais de saúde, pacientes e familiares auxilia no tratamento do câncer, na melhoria da QV e, em alguns casos, na condução de um modo de morrer mais digno.

\section{AGRADECIMENTOS}

Agradecemos ao CNPq pelo financiamento do projeto Condição de Saúde Bucal e QV em Pacientes com Câncer de Cabeça e Pescoço atendidos no SUS, através do Edital: MCT/SCTIE/DECIT/MS/CNPq no 038/2004 - Propostas sobre Saúde Bucal, do qual este trabalho faz parte.

\section{Potencial Conflito de Interesses: Nada a Declarar}

\section{REFERÊNCIAS}

1. De Haes J, et al. Quality of life evaluation in oncological clinical trials: the EORTC model. Eur J Cancer 2000; 36: 821-5.

2. Movsas B. Quality of life in oncology trials: a clinical guide. Semin Radiat Oncol 2003; 13 (3): 235-47.

3. Specht L. Oral complications in the head and neck radiation patient: introduction and scope of the problem. Support Care Cancer 2002; 10: 36-39.

4. Morton RP, Izzard ME. Quality of life outcomes in head and neck patients. World J Surg 2003; 27: 884-9.

5. Allison PJ, Locker D, Feine JS. The relationship between dental status and health-related quality of life in upper aerodigestive tract cancer patients. Oral Oncol 1999; 35: 138-43.

6. Rogers $\mathrm{SN}$, et al. Importance-rating using The University Of Washington quality of life questionnaire in patients treated by primary surgery for oral and oro-pharyngeal cancer. J Craniomaxillofac Surg 2002; 30: 125-32.

7. Neville BW. Complicações orais não-infecciosas do tratamento antineoplásico. In: Patologia oral e maxilofacial. 2. ed. Rio de Janeiro: Guanabara Koogan; 2004. p.251-5. 
8. De Graeff, et al. A prospective study on quality of life of patients with cancer of the oral cavity or oropharynx treated with surgery with or without radiotherapy. Oral Oncol 1999; 35: 27-32.

9. European Organization for Research and Treatment of Cancer (EORTC) [cited 2003 Dec 15]. Available from: <http://www.eortc.be/home/qol>

10. Organização Mundial de Saúde (OMS). Levantamentos básicos em saúde bucal. 4. ed. São Paulo, 1999.

11. Sreebny LM, Valdini A, Brook S. Xerostomia. Part I: Relationship to other oral symptoms and salivary gland hypofunction. Oral Surg Oral Med Oral Pathol 1988; 66: 451-8.

12. Sociedade Brasileira para Estudo da Dor. Dor: diagnóstico da dor. São Paulo, [2004]. [acesso em mai 2004] Disponível em: <http://www.dor.org.br/dor_diagnosticos.asp>

13. Baker C. Factors associated with rehabilitation in head and neck cancer. Cancer Nurs 1992; 15 (6): 395-400.

14. Fayers PM, et al. The EORTC QLQ-C 30 Scoring Manual. 3. ed. Bruxelas: European Organization for Research and Treatment of Cancer; 2001.

15. Bittencourt R, Scaletzky A, Boehl JAR. Perfil epidemiológico do câncer na rede pública em Porto Alegre - RS. Revista brasileira de cancerologia 2004; 50 (2): 95-101.

16. Kowalski LP, Nishimoto IN. Epidemiologia do câncer de boca. In: Parise Junior O. Câncer de boca: aspectos básicos e terapêuticos. São Paulo: Sarvier; 2000. p.3-11.

17. Hossfeld DK, et al, editores. Manual de oncologia clínica. 5. ed. São Paulo: Fundação Oncocentro de São Paulo; 1989.
18. Nicolatou-Galitis O, et al. Oral candidiasis in head and neck cancer patients receiving radiotherapy with amifostine cytoprotection. Oral Oncol 2003; 39 (4): 397-401.

19. Brasil. Ministério da Saúde. Secretaria de Atenção à Saúde. Departamento de Atenção Básica. Coordenação Nacional de Saúde Bucal. Projeto SB Brasil 2003: condições de saúde bucal da população brasileira 2002-2003: resultados principais. Brasília, 2004b. [acesso em abr 2004] Disponível em: <http://www.cfo.org.br/download/pdf/ relatorio_sb_brasil_2003.pdf>.

20. De Graeff, et al. Head and neck cancer. In: Fayers P, Weeden S, Desmond C. EORTC QLQ-C 30 reference values. Bruxelas: European Organization for Research and Treatment of Cancer; 1998. p. 23-47.

21. Eisbruch A, et al. How should we measure and report radiotherapy-induced xerostomia? Semin Radiat Oncol 2003; 13 (3): 226-34.

22. Chen M, Harmon P, Andersen R. Oral quality of life. In: Chen $\mathrm{M}$, et al. Comparing oral health care systems: a second international collaborative study. Geneva: World Health Organization: University of Chicago Center for Health Administration Studies; 1997. p. 187-96.

23. Rogers SN. Reflections on psychology and head and neck cancer. Br J Oral Maxillofac Surg 2001; 39: 346.

24. Rose-Ped AM, et al. Complications of radiation therapy for head and neck cancers: the patient's perspective. Cancer Nurs 2002; 25 (6): 461-7.

25. Bjordal K, etal. Patients self-reported and clinician-rated quality of life in head and neck cancer patients: a cross-sectional study. Eur J Cancer B Oral Oncol 31B (4): 235-41.

\section{Abstract}

The aim of the present study was to evaluate the QOL (Quality of Life) in patients suffering from head and neck cancer related to oral health conditions. Several social and economic factors and habits were analyzed, including aspects related to tumor and oral health conditions - DMF-T (Caries Index is constituted by decayed teeth, missing teeth and tooth fillings), uses the need of prosthesis, xerostomia, and dentist appointments in the previous six months, mucositis and curtailment. Two questionnaires - Quality of Life Questionnaire - Cancer30_Portuguese Brazilian, version 3.0 and EORTC Quality of Life Questionnaire - Head\&Neck35_Portuguese Brazilian - were used. At first, the QOL was considered as median, with a moderate functional level and low symptomatology. There was no significant statistic correlation between lost and decayed components of the DMF-T with QOL. The group of patients who needed prostheses reported minor performance and social function. The group of patients with xerostomia showed a lower QOL, with a lower level of functioning and higher symptomatology. During the follow up, patients showed no QOL changes when compared to the first period of the study; however, the symptoms increased and the function level decreased. The CPOD increased and the use of prosthesis decreased, whereas the need of it increased. It was not possible to verify the relationship between QOL and mucositis, as only few patients were affected by this condition. Curtailment was negatively connected to the performance function, nausea, vomiting and appetite loss. Clinic evaluation of oral health alone was not enough to establish its connections with QOL. Nevertheless a dentist must be part of the multidisciplinary oncology staff to minimize the damage to the patient's oral health, in order to increase the patients QOL.

Key words: Quality of life; Oral health; Head and neck neoplasms; Epidemiology 